\title{
Cyclophosphamide Induced Histologial and Immunohistochemical Alterations in Kidney of Albino Rats: The Ameliorative Effect of Fennel Oil
}

\author{
Saber A. Sakr ${ }^{1}$, Ferial A. El-messady ${ }^{1}$
}

${ }^{1}$ Zoology department, Faculty of Science, Menoufia University, Egypt

\begin{abstract}
Cyclophosphamide (CPA) is a nitrogen mustard alkylating agent from the oxazaphosphorine group. It is used as an anticancer, anti-neoplastic agents and immunosuppressive agent widely used in chemotherapy. Fennel oil is traditionally used as a treatment for different diseases. The present work studied the effect of Fennel oil on CPAinduced nephrotoxicity in albino rats. Male albino rats were divided into 4 groups; gp1; control, gp2: Each rat was orally given Fennel oil ( $1 \mathrm{ml} / \mathrm{kg}$ body weight once a week for six weeks), gp3: animals were each orally treated with CPA at a dose of $15 \mathrm{mg} / \mathrm{kg}$ body weight once a week for six weeks, gp4: CPA plus Fennel oil treated group. The obtained results showed that CPA induced histopathological alterations in the kidney including intertubular leucocytic infiltrations,congestion of blood vessels, degeneration of renal tubules and atrophy of glomeruli. Immunohistochemical observations revealed increase in expression of PCNA, caspase- 3 and $\alpha$-SMA. In addition, creatinine and urea levels increased in sera of treated rats. Treating rats with CPA and fennel oil caused an improvement in the histological structure of the kidney and decreased PCNA, caspase- 3 and $\alpha$-SMA. Moreover, creatinine and urea values were decreased. In conclusion, fennel oil showed ameliorative effect against nephrotoxicity of CPA due to its antioxidant activity.
\end{abstract}

Keywords: Cyclophosphamide, Fennel Oil, Kidney, Histology, ,PCNA, $\alpha$-SMA, Rats

\section{Introduction}

Recent studies on therapy of diseases have focused on the ideal drug being ineffective or minimally effective for normal cells. At this point, the usage of natural sources is thought to have a great value for treating diseases. Moreover, there is a growing trend for herbal drugs because of low toxicity and high medical effectiveness. Fennel is a medicinal plant belongs to the family Apiaceae (Umbelliferae) [1]. This herb is traditionally used as treatment for colic, wind, irritable bowel, kidneys, spleen, liver, lungs, suppressing appetite, breast enlargement, promoting menstruation, improving digestive system, milk flow and increasing urine flow [2]. The chief component of fennel, anethole, had anticarcinogenic and antiinflammatory effects through modulation tumor necrosis factor-induced cellular processes [3]. Essential oils of fennel have hepatoprotective effects [4], antifungal effects [5],antimicrobial prosperities [6] as well as anti-inflammatory, and antioxidant activities [7].

Cyclophosphamide is a nitrogen mustard alkylating agent from the oxazaphosphorine group. It is used as an anticancer, anti-neoplastic agents and immunosuppressive agent widely used in cytotoxic chemotherapy, which disturbs DNA synthesis and cell division [8]. Cyclophosphamide is rapidly absorbed and then converted by mixed-function oxidase enzymes (cytochrome P450 system) in the liver to active metabolites [9]. The main active metabolite is 4-hydroxycyclophosphamide, which exists in equilibrium with its tautomer, aldophosphamide. Most of the aldophosphamide is then oxidised by the enzyme aldehyde dehydrogenase (ALDH) to make carboxycyclophosphamide. A small proportion of aldophosphamide freely diffuses into cells, where it is decomposed into two compounds, phosphoramide mustard and acrolein [10]. However, acrolein is a highly reactive aldehyde that covalently binds to cellular macromolecules and subsequently disrupts the function and causes organ toxicity [11]. Cyclophosphamide is carcinogenic and may increase the risk of developing lymphomas, leukemia, skin cancer, transitional cell carcinoma of the bladder or other malignancies [12]. Cardiotoxicity is a major problem with people treated with higher dose regimens [13]. In addition, toxicity of

This article is published under the terms of the Creative Commons Attribution License 4.0

Author(s) retain the copyright of this article. Publication rights with Alkhaer Publications.

Published at: http://www.ijsciences.com/pub/issue/2017-08/

DOI: 10.18483/ijSci.1383; Online ISSN: 2305-3925; Print ISSN: 2410-4477 
cyclophosphamide was report in liver [14]. kidney [15] and testis [16]. The present work investigated the effect of fennel oil on nephrotoxicity of CPA in male albino rats.

\section{Materials and methods \\ i.Animals and treatments:}

Adult male albino rats (Rattus norvigicus), three months age weighting $150 \pm 10 \mathrm{~g}$ were purchased from experimental rat house localized in Helwan. Animals were kept in plastic cages (each contained six animals) in the animal house for two weeks before the experimental work. Animals were kept at $25 \pm 2$ $\mathrm{C}$ with relative humidity of $50-60 \%$ and on $12 \mathrm{~h}$ light/ $12 \mathrm{~h}$ dark cycle. They received a standard diet composed of $50 \%$ barley, $20 \%$ yellow corn, $20 \%$ dry milk, $10 \%$ different vegetables and tap water. The study and all procedures were approved by the Animal Care and Bioethics Committee, Menoufia University, Egypt (Approval No. MNSH174). Animals were divided into four groups

Group 1 (Control group): Animals of this group (10 rats) were served as control group and were given standard diet and tap water.

Group 2 (Fennel oil group): Animals of this group (12 rats) were orally given fennel oil at a dose level of $1 \mathrm{ml} / \mathrm{kg}$ body weight once a week for six weeks [17]. Fennel essential oil was purchased from a local market at shebin El-Kom , Menufyia , (El Masry Everline company).

Group 3 (CPA group):Animals of this group (12 rats) were orally treated with endoxan at a dose of $15 \mathrm{mg} / \mathrm{kg}$ body weight once a week for six weeks [18. Endoxan was obtained as tablets from Baxter Oncology Halle, Germany. It was dissolved in distilled water and orally given by gastric tube.

Group 4 (CPA+Fennel oil group): Animals were given endoxan and then they were given fennel oil, with the same doses of group 2 and 3 .

\section{ii.Histological studies:}

For histological study, kidneys were immediately removed after 6 weeks, and fixed in 10\% formalin for 24 hours. Specimens were dehydrated in ascending series of ethyl alcohol, cleared in two changes of xylene, infiltrated in three changes of molten paraffin (melting point $58-60{ }^{\circ} \mathrm{C}$ ) and then embedded in molten paraffin blocks. Paraffin sections (5 micron thickness) were sectioned using a rotary microtome and mounted on clean glass slides. Sections were stained with Ehrlich's hematoxylin and counter stained with eosin for histological examination.

\section{E) Immunohistochemical studies:}

Immunohistochemical reaction was performed by using an avidin biotin complex immune peroxidase technique. For Immunostaining of PCNA, Caspase 3 and $\alpha$-SMA, formalin-fixed slides were deparaffinized in xylene and rehydrated in a series of graded alcohol concentrations. Then rinsed in phosphate-buffered saline (PBS) containing $0.1 \%$ tween-20. Antigen retrival was performed by placing slides in sodium citrate solution $(\mathrm{PH} 6.0)$ at $90^{\circ} \mathrm{C}$. Avidin $(0.001 \%$ in PBS) and biotin $(0.001 \%$ in PBS) were blocked in each section by using Avidin/biotin blocking solutions, where sections were incubated and rinsed with PBS between steps. Sections were incubated with monoclonal primary rat antibodies (Neo Markers, Cat.\#Ms-113-P, Fremont, CA,USA), at appropriate dilution $(1: 200)$ in antibody diluent, directed against rat PCNA, Caspase 3 and $\alpha$-SMA (each antibody was used separately to react on different slides) at room temperature. Slides were washed in PBS-Tween 20. Sections were incubated in peroxidase blocking solution $\left(3 \% \mathrm{H}_{2} \mathrm{O}_{2}\right.$ in PBS) at room temperature. Slides were washed in PBSTween 20. Sections were incubated in biotinylated secondary antibody in PBS at room temperature. Slides were washed in PBS-Tween 20. Sections were incubated in horse radish peroxidase (HRP)streptavidin solution at room temperature. Slides were washed in PBS-Tween 20. Sections were incubated in peroxidase substrate solution "3,3diaminobenzidine tetrahydrochloride (DAP)" until adequate color was developed. Slides were washed in PBS-Tween 20. Sections were counterstained with hematoxylin, dehydrated through garded alcohol series, clear in xylene and mounted with DPX [19].

\section{Image analysis}

Digital images were analyzed by a semiquantitative scoring system (Image J software, Java based application for analyzing images). The positivestained immunohistochemical expressions of PCNA, Caspase 3 and $\alpha$-SMA in cells were measured by counting the number of positively- stained cells in five high power fields selected randomly at magnification X400.

\section{F- Biochemical analysis:}

For biochemical analysis, blood samples were collected in clean centrifuge tubes. Blood samples left to clot in room temperature and then serum separated by centrifugation at $3000 \mathrm{rpm}$ for 20 minutes. The collected serum stored at $-18-20{ }^{\circ} \mathrm{C}$ until analysis. Creatinine and urea were estimated using the methods described by Henry [20] and Patton and Crouch [21] respectively.

\section{G- Statistical Analysis}

Data were expressed as mean \pm standard deviation (SD). The significance of differences means was evaluated by using independent sample $t$ test. All statistical analysis was performed using SPSS statistical version 16 software package. 


\section{Results}

\section{Histological results}

Microscopic examination of kidney of control rat showed normal structure of the renal cortex, which comprised renal corpuscles and proximal and distal convoluted tubules (Fig.1A). No histological alterations were observed in rats given fennel oil (Fig.1B). On the other hand, kidney sections of animals treated with CPA revealed different histological changes. The renal veins were enlarged and congested (Fig.1C) and leucocytic infiltrations were abundant in the interstitum (Fig.1D). The renal tubules were degenerated and their lumens were filled with proteinaceous casts (Figs.2A). The renal tubules were degenerated and their epithelial cells appeared with cytoplasmic vacuolation (Fiig.2 B). The glomeruli were atrophied with dilatation in the subcapsular space (Fig.2C). Animals treated with CPA and fennel oil showed an improvement in the structural elements of the kidney and most of the renal tubules and the glomeruli appeared normal (Fig.2D).

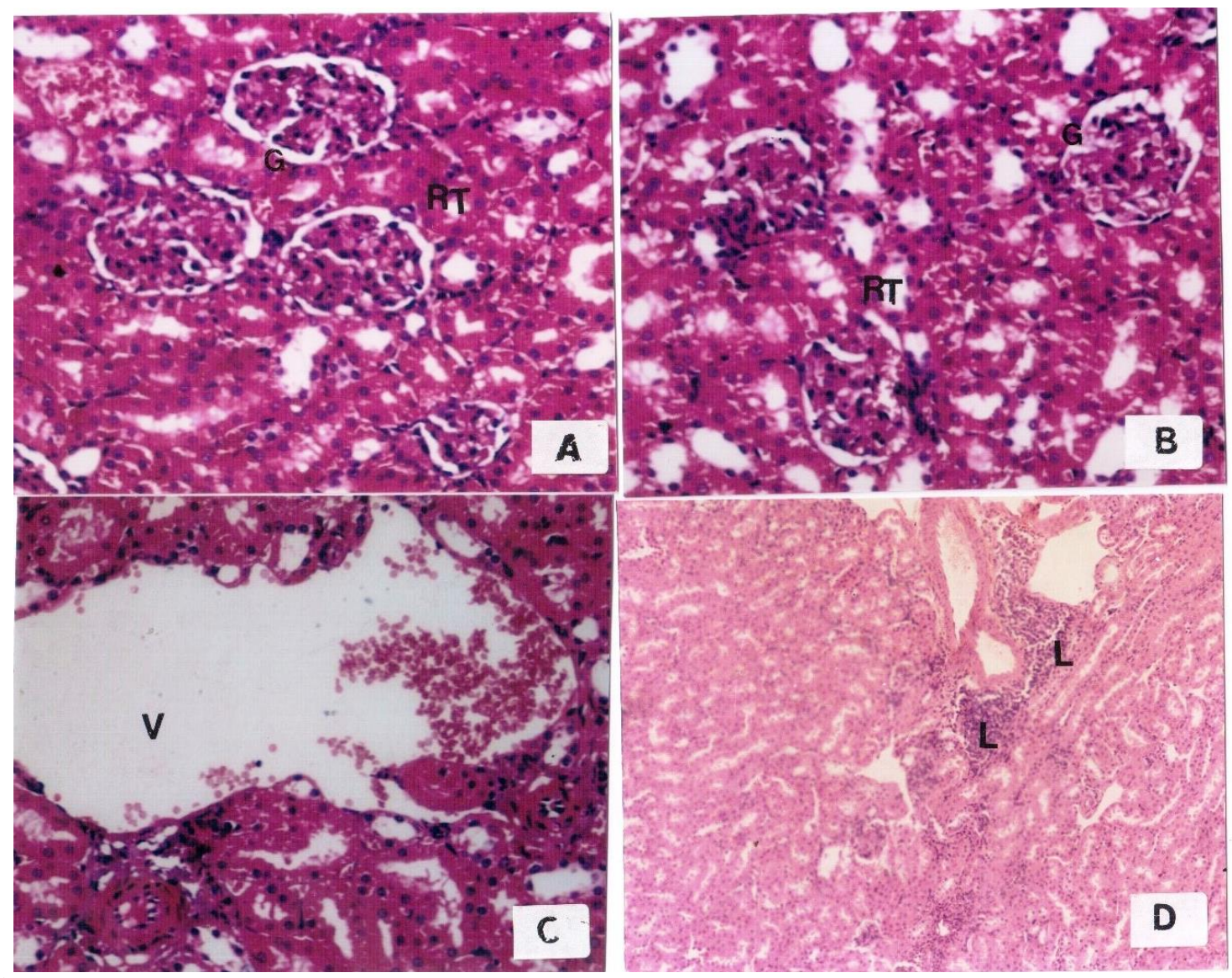

Fig.1:

A. Section in Kidney cortex of a control rat showing normal-structured renal tubules (RT) and glomeruli (G) (X400).

B. Kidney cortex of fennel oil -treated rat showing normal structure, (X400).

C. Section in kidney cortex of a rat treated with CPA showing enlarged and congested renal vein (v) (X400).

D. Interstitial leucocytic infiltrations (L), (X100) 
Fig.2:

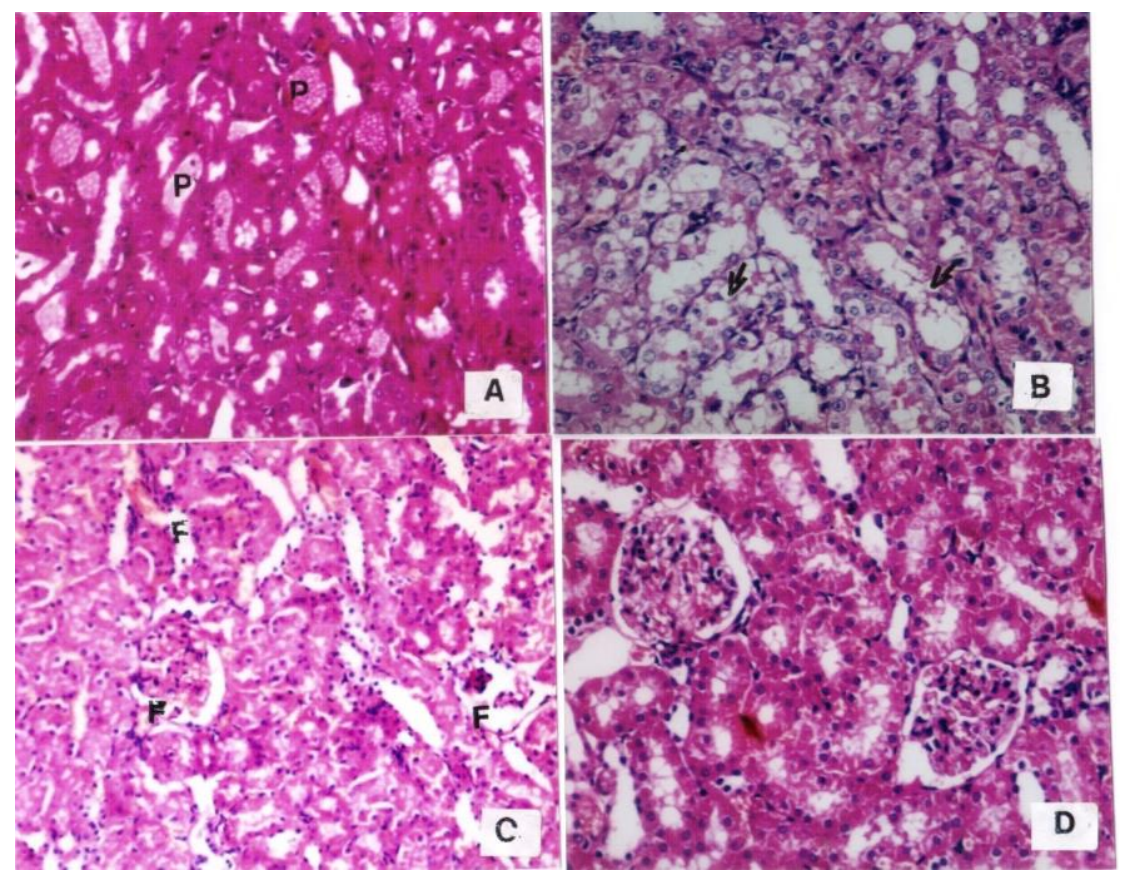

A. Kidney cortex of CPA-treated rat showing degenerated tubules with with lumen filled with proteinous casts (P), (X400).

B. Highly degenerated tubules with cytoplasmic vacuolation of epithelial cells, (X400).

C. Fragmented glomeruli (F), (X400).

D. Kidney cortex of a rat treated with $\mathrm{CPA}+$ fennel oil showing an improvement in the structure of the tubules and glomeruli, (X400).

\section{Immunohistochemical observations}

\section{PCNA:}

Examination of kidney of control rats and those given fennel oil showed few expression of PCNA in the nuclei (Fig.3.A,B). A strong expression of PCNA was recorded in renal tubules of animals treated with
CPA(Fig.3C). Treating rats with CPA and fennel oil led to a decrease of expression of PCNA (Fig.3D). Image analysis of PCNA immunoreactivity in CPAtreated animals resulted in a significant increase $(\mathrm{P} \leq$ $0.05)$ as compared to that of controls. However, fennel oil lowered PCNA expression as compared to that of CPA alone (Fig.4).
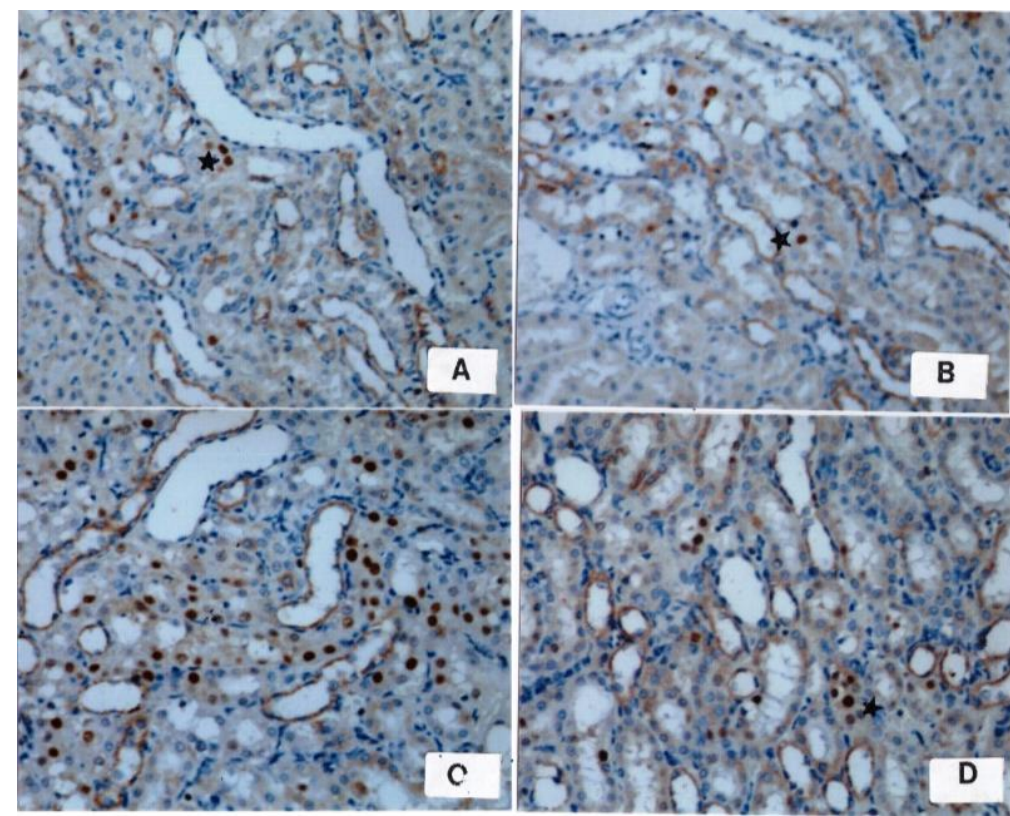

Fig.3. 
A. Section of kidney cortex of a control rat showing expression of PCNA in the nuclei of few cells of renal tubules

(*) (PCNA immunohistochemical stain, X400)

B. Section of kidney of fennel oil treated rat showing expression of PCNA in the epithelia of few renal tubules (*) (PCNA immunohistochemical stain, X400)

C. Kidney cortex of a rat treated with CPA showing strong expression of PCNA in the epithelia of renal tubules (PCNA immunohistochemical stain, X400).

D. Section of kidney of a rat treated with CPA and fennel oil showing weak expression of PCNA in the epithelia of renal tubules $(*)$ (PCNA immunohistochemical stain, X400).

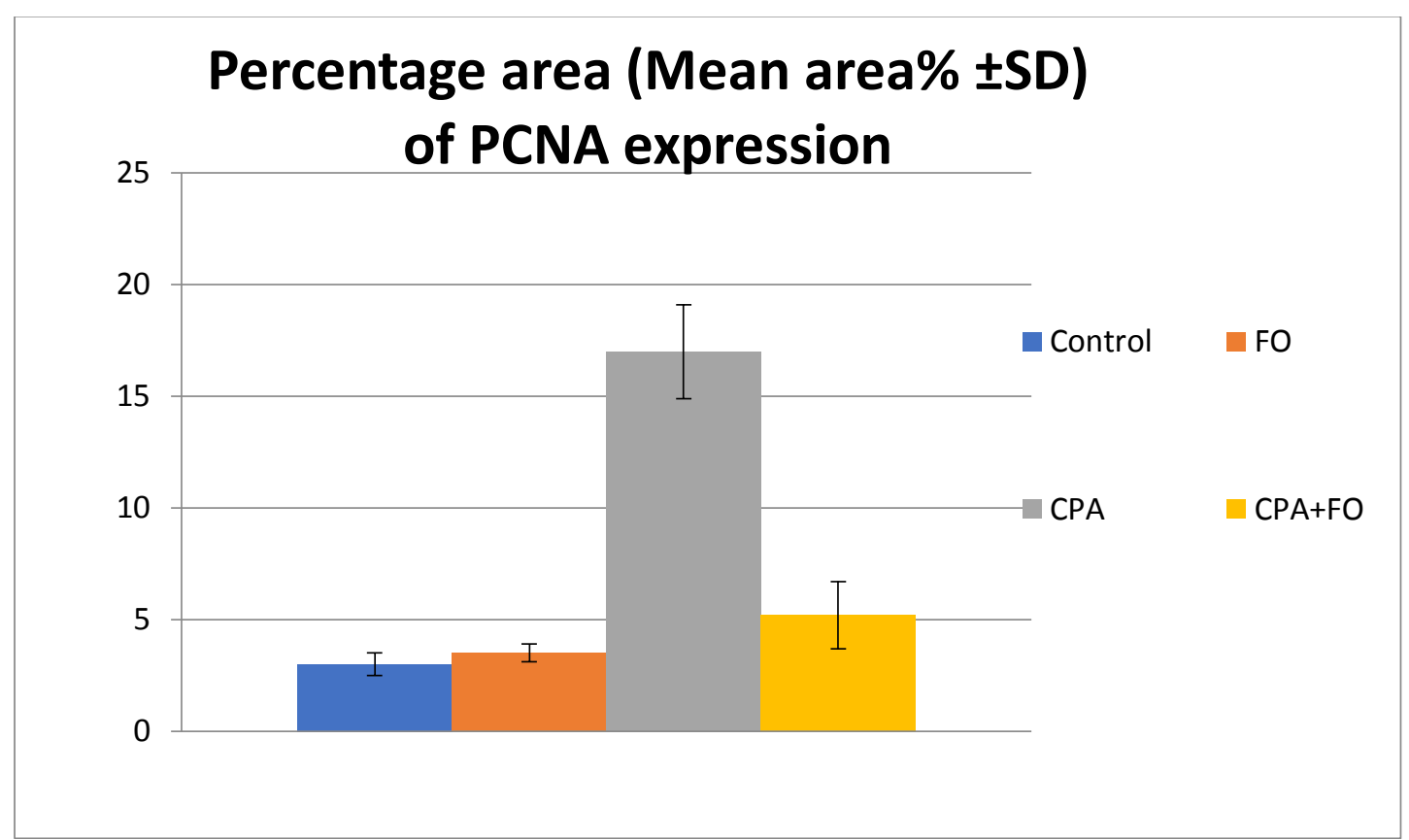

Fig.4. Percentage area of PCNA expression in different animal groups.

\section{Caspase 3:}

Kidney sections of control rats showed weak brown staining of Caspase 3 expression in the cytoplasm of renal epithelia (Fig.5A). Similarly, renal tubules of rats given fennel oil showed weak expression of Caspase 3 (Fig. 5B). Strong immunoreactions was encountered in cytoplasm of large number of epithelial cells of renal tubules of animals treated with CPA (Fig.5C). Animals treated with CPA and fennel oil showed a decrease in expression of caspase-3 (Fig.5D). By image analysis, the reaction of Caspase 3 in kidney recorded a significant elevation in CPA-treated animals .A decrease in expression of Caspase 3 was observed in rats treated with CPA and fennel oil (Fig.6).
a-SMA:

Examination of kidney cortex of control animals and animals given fennel oil showed that $\alpha$-SMA was expressed in the smooth muscle cells of renal arterioles (Fig. 7A,B). Rats treated with CPA showed an increase in the expression of $\alpha$-SMA (Fig. 7C). Animals treated with CPA and fennel oil showed reduction in expression of $\alpha$-SMA compared to treatment with CPA alone (Fig. 7D). Image analysis of $\alpha$-SMA expression in renal tissue showed that the percentage of $\alpha$-SMA-positive staining area significantly $(\mathrm{P}<0.05)$ decreased in the $\mathrm{CPA}$ and fennel oil group compared with CPA group (Fig. 8). 


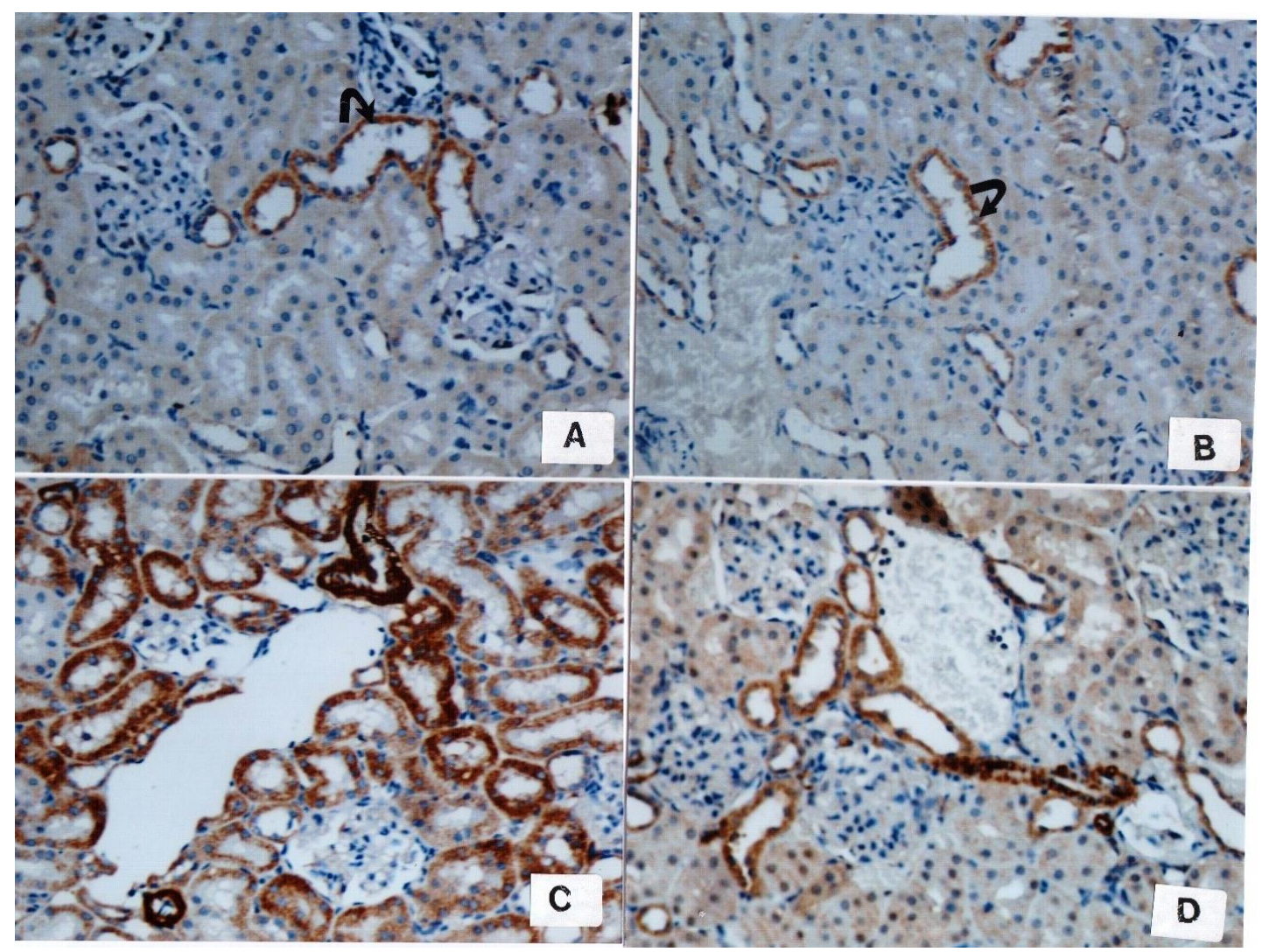

Fig.5.

A.Section of kidney of a control rat showing expression of caspase-3 in the epithelia of few renal tubules (arrow) (caspase-3 immunohistochemical stain, X400)

B. Section of kidney of fennel oil treated rat showing positive expression of caspase-3 in the epithelia of few renal tubules (arrow) (caspase-3 immunohistochemical stain, X400)

C. Kidney cortex of a rat treated with CPA showing strong expression of caspase-3 in the epithelia of renal tubules (caspase-3 immunohistochemical stain, X400).

D. Section of kidney of a rat treated with CPA and fennel oil showing weak expression of caspase-3 in the epithelia of renal tubules (caspase-3 immunohistochemical stain, X400).

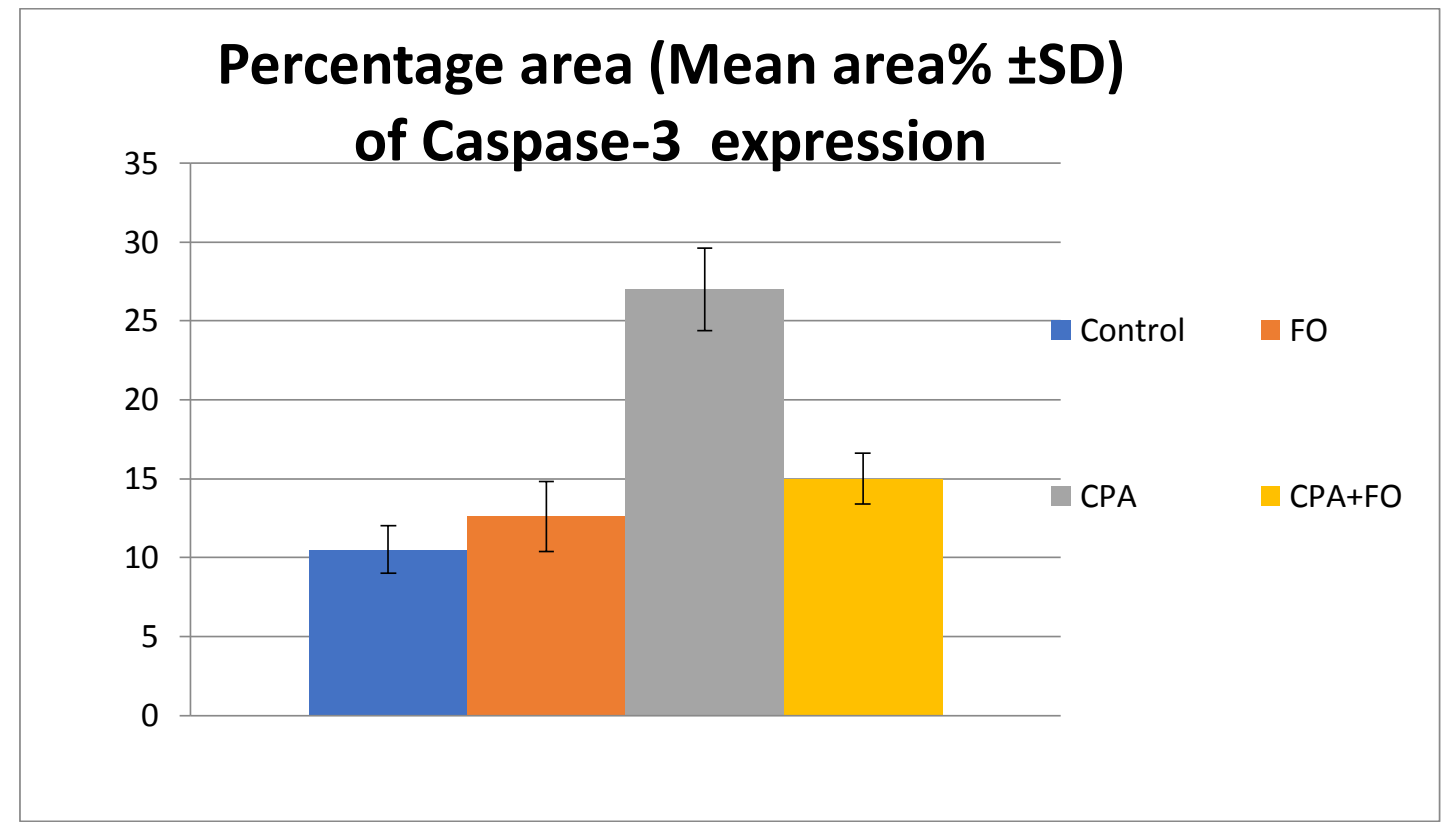

Fig.6. Percentage area of caspase-3 expression in different animal groups. 


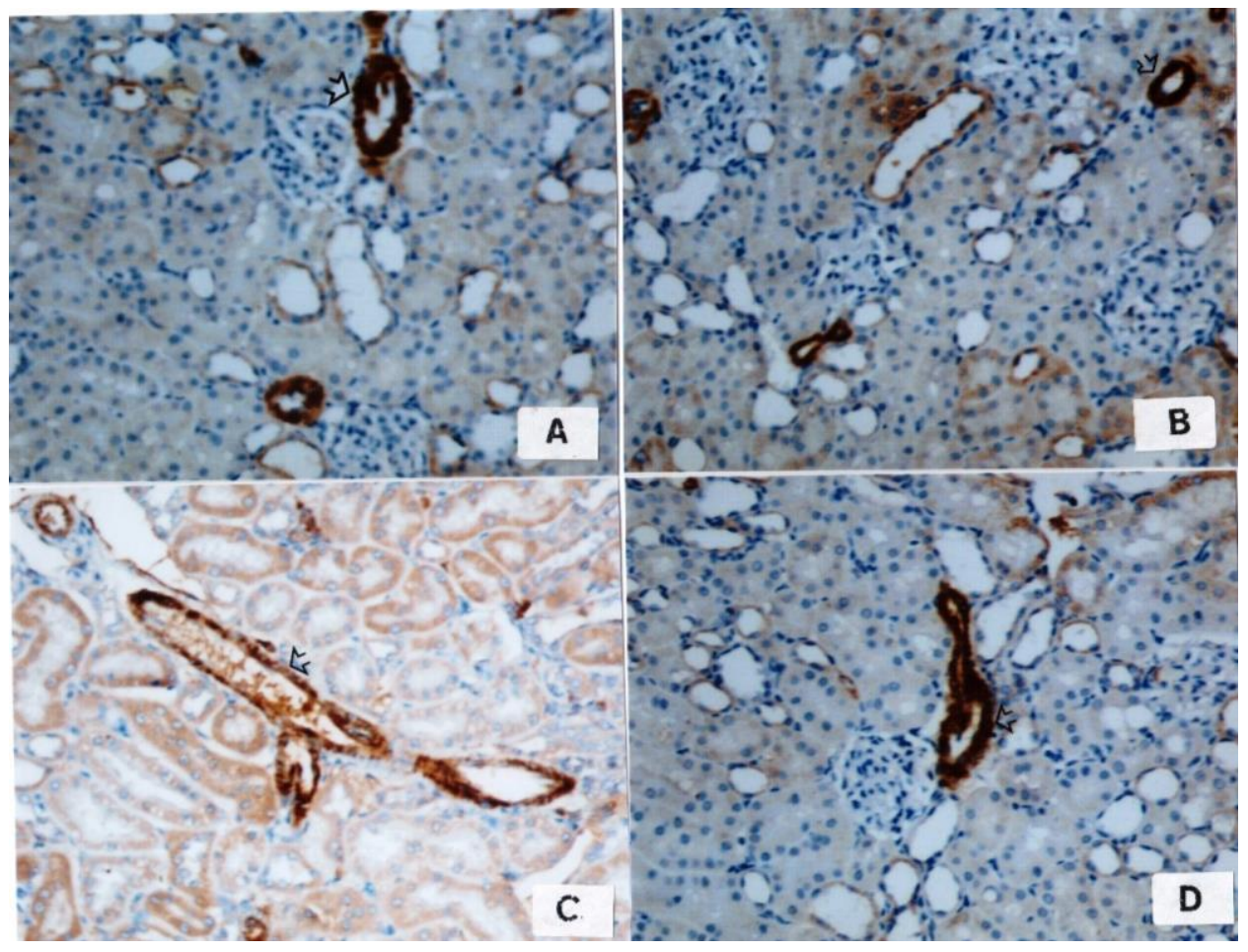

Fig. 7.

A. Section in kidney cortex of a control rat showing $\alpha$-SMA immune- staining in smooth muscle of renal arterioles (arrow head) (X400).

B. Section in kidney cortex of a rat treated with fennel oil showing expression of $\alpha$-SMA in smooth muscle of renal arterioles (arrow head) (X400).

C. Section of kidney cortex of CPA treated rat showing an increase in expression of $\alpha$-SMA in renal tubules (Arrow head) (X400).

D. Section of kidney cortex of CPA+fennel oil treated rat showing decrease in expression of $\alpha$-SMA positive fibroblastic renal cells (X400).

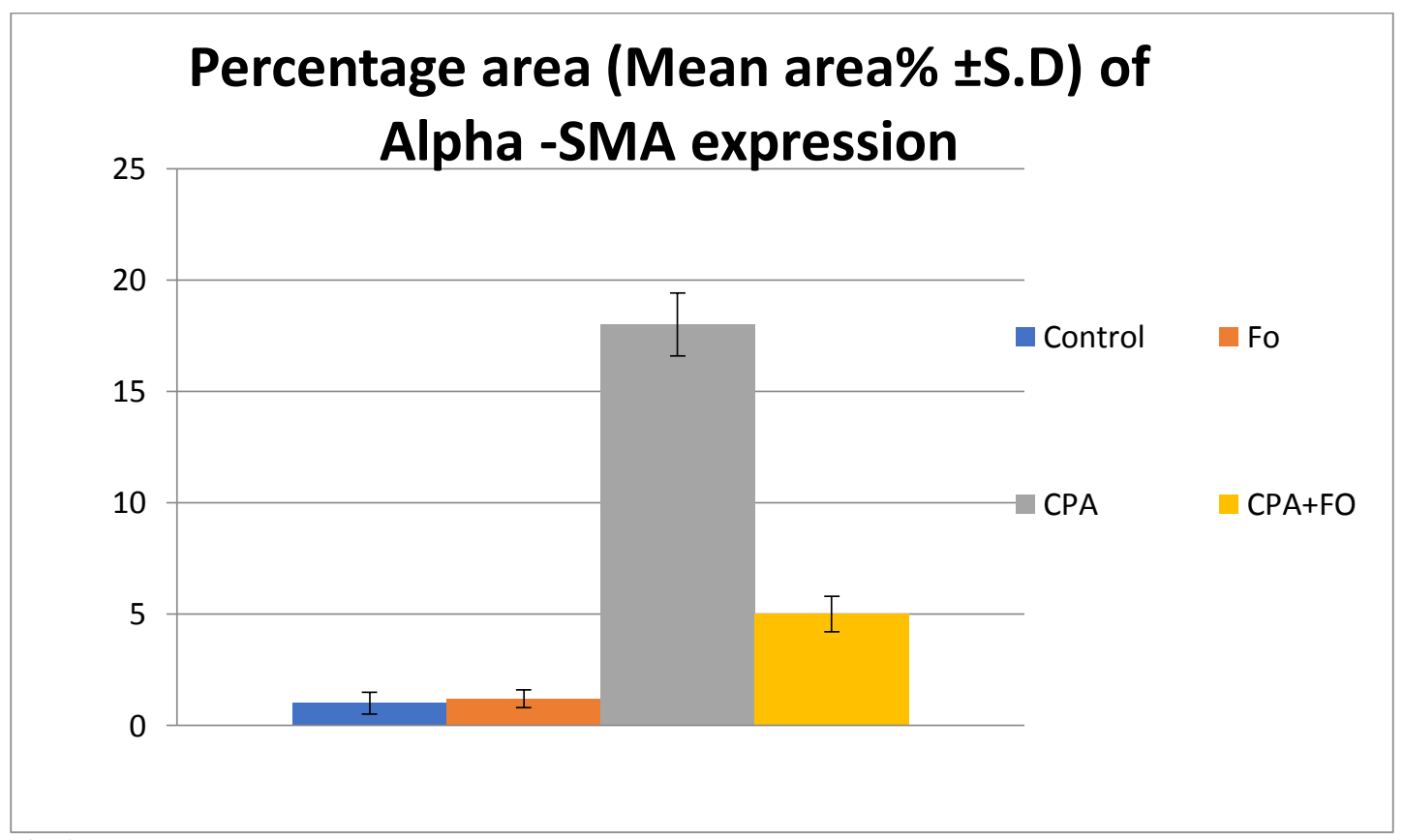

Fig.8. Percentage area of $\alpha$-SMA expression in different animal groups. 


\section{Biochemical results:}

Treating animals with CPA caused significant increase $(\mathrm{P}<0.05)$ in serum level of creatinine and urea nitrogen compared with controls and those given fennel oil (Fig.9\&10). On the other hand rats treated with CPA and funnel oil recorded a decrease in the level of creatinine and urea compared with CPA group.

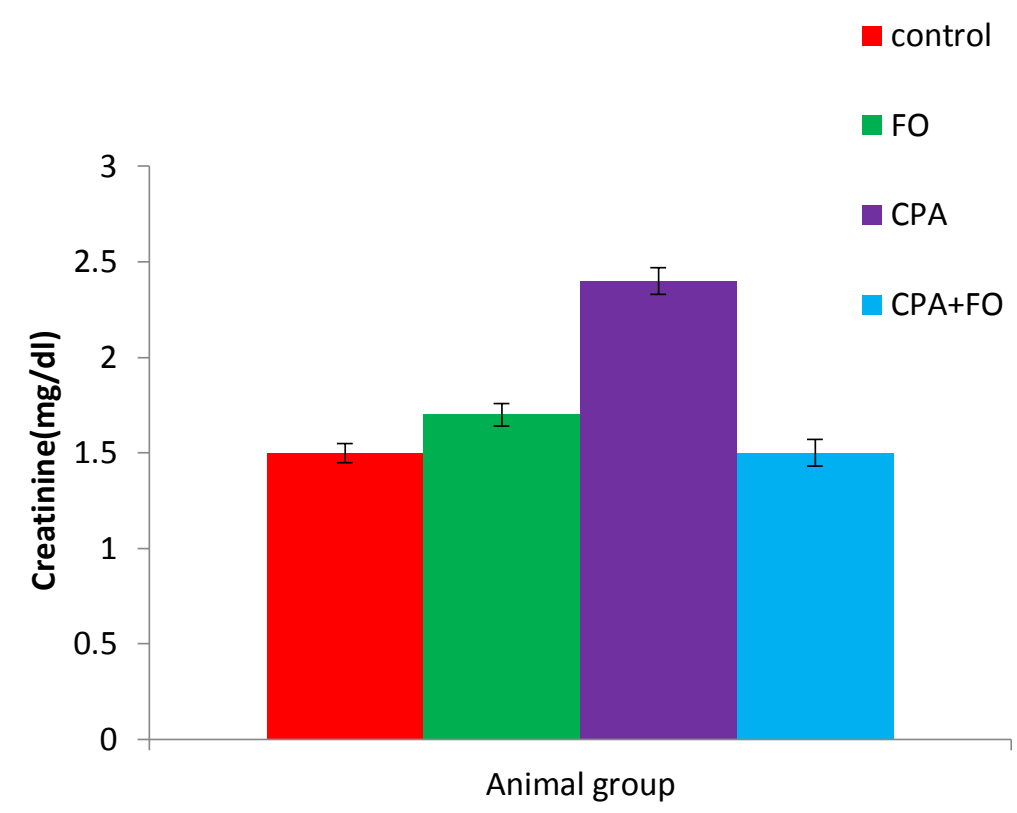

Fig.9. Effect of different treatments on creatinine

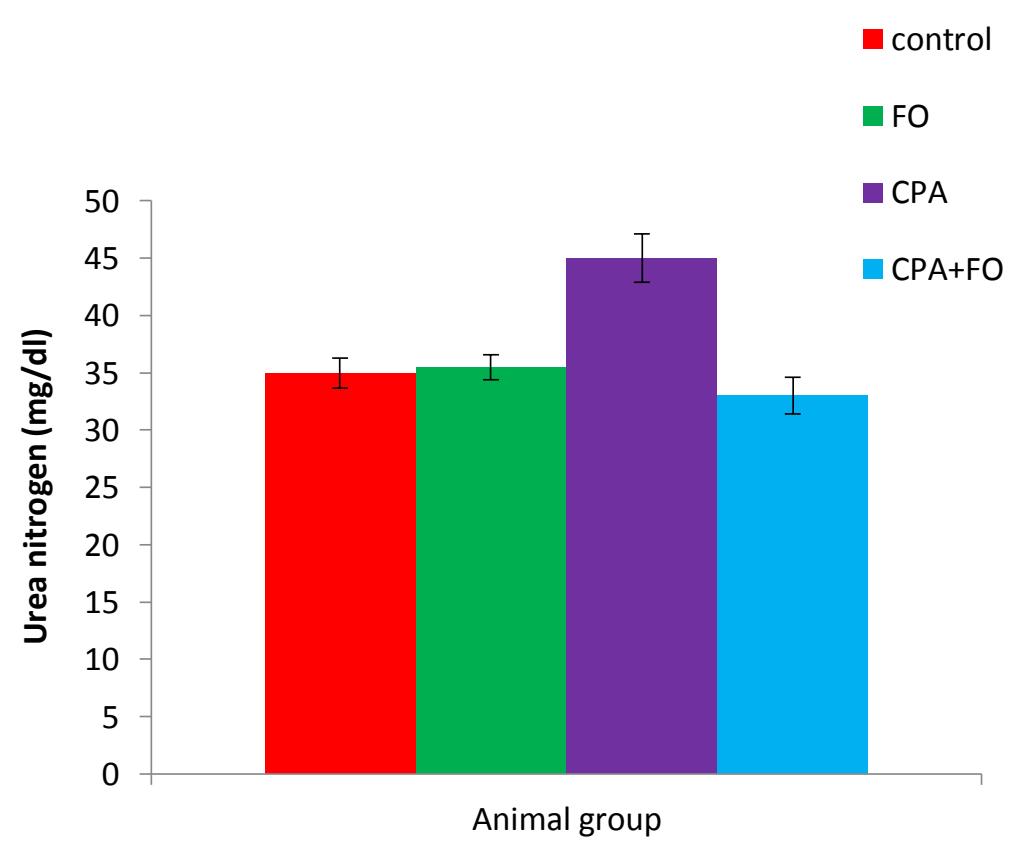

Fig.10. Effect of different treatments on urea nitrogen

\section{Discussion}

Cyclophosphamide is an anticancer drug used in treatment of many neoplastic diseases. On the other hand, the use of CPA was accompanied with different deleterious effects. Results obtained in the present work revealed that CPA induced histopathological alterations in the kidney of rats. In addition, it caused elevation in levels of creatinine and 
urea.Similarly,Sakr and abdel-samei [15] reported that CPA administration induced many histological alterations in the kidney including congestion of renal blood vessels, leucocytic infiltrations and degeneration of renal tubules as well as atrophy of glomeruli. An elevation in creatinine and urea was also recorded. They added that Ginko biloba extract improved these deleterious effects. Estakhri et al. [22] observed a significant increase in creatinine and blood urea nitrogen levels and histopathologic change in renal tissue upon CPA treatment. Abraham and Rabi [23] reported reduction of glomerular filtration rate along with tubular dysfunction, glomerular nephritis, interstitial edema and cortical tubular vacuolization in rats treated with CPA.

Immunohistochemical results revealed that rats treated with CPA showed an increase in the expression of PCNA, caspase 3 and $\alpha$-SMA. PCNA is an auxiliary protein of DNA polymerase delta and is involved in a wide range of functions in the nucleus such as control of DNA replication, cell-cycle progression, transcription as well as DNA damage repair [24]. Urothelium of rats treated with CPA was found to have elevated expression of PCNA [25]. Apoptosis plays a complementary and opposing role to cell proliferation in regulating the number of cells in tissues [26]. Caspase-3 is a marker of the early phase of apoptosis and is essential for certain processes associated with the formation of apoptotic bodies [27]. In agreement of our results, CPA treated rats showed significant increase in expression of the apoptotic marker casepase-3 in liver [28]. An increase in $\alpha$-SMA expression in kidney of CPAtreated rats was recorded in the present work. Similar result was obtained by Sakr and Abdel-samei [15]. The increase in expression of $\alpha$-SMA was suggested to an evidence for activation of fibrogenesis during glomerulonephritis [29].

Phosphoramide mustard and acrolein are the two active metabolites of cyclophosphamide[30]. Cyclophosphamide's antineoplastic effects are associated with the phosphoramide mustard, while acrolein is linked with its toxic effects [31]. Acrolein interferes with the tissue antioxidant defense system, produces highly reactive oxygen free radicals and is mutagenic to mammalian cells [32]. It was reported that treatment with CPA caused oxidative stress in different organs including brain [33], liver [34] and kidney [15]. Thus, the nephrotoxicity recorded in the current study may be attributed to oxidative stress induced by CPA.

Treating rats with fennel oil protects the kidney from the toxicity of CPA. Combined administration with fennel oil and CPA improved the histological and immunohistochemical alterations, and restored the values of creatinine and urea. In agreement with this result, Al-masri and Ali [35] reported that fennel powder and oil ameliorated gentamicin-induced nephrotoxicity in rats and reduced the elevation in urea and creatinine. Al-amoudi [36] showed that treating with fennel oil prevent the histopathological alterations in liver and kidney, and decreased levels of urea and creatinine caused by sod valporate in rats. Fennel oil administration led to a decrease in expression of PCNA and Caspase- 3 in te kidney of rats treated with CPA. These results indicated the antiproliferative and antiapoptotic effects of fennel oil. Similarly, Ibrahim [37] reported that fennel oil decreased apoptosis in testes of rats exposed to tobacco smoke. In addition, $\alpha$-SMA expression was reduced in the kidney which means the antifibrotic effect of fennel oil.

Fennel oil contains different compounds including trans-anethole (1-methoxy-4-(1- propenyl) benzene or para-propenylanisole), fenchone and estragole.It has physiologic antioxidant activities including the radical scavenging effect, inhibition of hydrogen peroxides $\mathrm{H}_{2} \mathrm{O}_{2}$ and $\mathrm{Fe}$ chelating activities where it can minimize free radical which initiate the chain reactions of lipid peroxidation [38]. Sheweita et al. [39] found that fennel oil restored changes in activities of antioxidant enzymes SOD, CAT, GR, GST, and GPx caused by CPA to their normal levels compared to control mice. Sakr et al. [40] reported that treating rats with $\mathrm{CPA}$ and fennel oil caused an improvement in the histological structure of the liver, decreased ALT, AST, MDA and increased SOD and CAT. It is concluded from this work that fennel oil ameliorates nephrotoxicity of CPA by its antioxidant activity.

\section{Competing interests}

The authors declare that they have no competing interests

\section{References}

1. Rather, A.; Bilal A. Dar, Shahnawaz, Sofi, N.; Bilal A. Bhat, Mushtaq A. and Qurishi, A. (2016). Foeniculum vulgare: a comprehensive review of its traditional use, phytochemistry, pharmacology, and safety Arab. J. Chem.9(2): S1574-S1583

2. Delaram, M.; Kheiri, S. and Hodjati, M.R. (2011): Comparing the effects of echinop hora-platyloba, fennel and placebo on pre-menstrual syndrome J. Reprod. Infertil., 12 (3) : 221-226

3. Aggarwal, B. B.; Kunnumakkara, A. B.; Harikumar, K. B.; Tharakan, S. T.; Sung, B. and Anand, P. (2008): Potential of spice-derived phytochemicals for cancer prevention. Planta Med. 74(13): 1560-9.

4. Ozbek, H.; Uğraş, S.; Dülger, H.; Bayram, I.; Tuncer, I.; Oztürk, G. and Oztürk, A. (2003): Hepatoprotective effect of Foeniculum vulgare essential oil Fitoterapia, 74: 317-319

5. Mimica-Dukic, N., B. Bozin, M. Sokovic, B. Mihajlovic and M. Matavulj. (2003): Antimicrobial and antioxidant activities of three Mentha species essential oils. Planta Medica, 69: 413-419.

6. Soylu, E. M., Soylu, S., \& Kurt, S. (2006). Antimicrobial activities of the essential oils of various plants against tomato 
late blight disease agent Phytophthorainfestans. Mycopathologia, 161: 119-128.

7. Choi, E.M. and Hwag, J.K. (2004): Anti-inflammatory, analgesic and antioxidant activities of the fruit of Foeniculum vulgare . Fitoterapia. 75(6): 557-565

8. Takimoto, CH. \& Calvo, E. (2005): Principles of oncologic pharmacotherapy. In: Cancer Management: A Multidisciplinary Approach. Medical, Surgical \& Radiation Oncology. Pazdur, R.; Coia, LR.; Hoskins WJ \& Wagman, LD. (Eds.), pp. 23-43, CMP Healthcare Media, Washington, USA

9. Huttunen, K.M.; Raunio, H. and Rautio, J. (2011): Prodrugs-from serendipity to rational design. Pharmacological Reviews 63 (3): 750-71

10. Boddy Av and Yule SM (2000): Metabolism and pharmacokinetics of oxazaphosphorines . Clin Pharmacokinet 38(4) : 291-304

11. Kehrer JP, Biswal SS.(2000): The molecular effects of acrolein. Toxicological Sciences. 57:6-15.

12. Bernatsky S, Clarke AE.(2008): Ramsey-Goldman R. Cancer in systemic lupus: what drives the risk? Cancer Causes Control CCC. 19(10):1413-1414

13. Floyd, J.D.; Nguyen, D.T.; Lobins, R.L.; Bashir, Q.; Doll, D.C. and Perry, M.C. (2005): Cardiotoxicity of cancer therapy. J. Clinical Oncology 23 (30): 7685-96

14. Li, F.; Patterson, A.D., Höfer, C.C.; Krausz, K.W.; Gonzalez, F.J. and Idle, J.R.(2010): Comparative metabolism of cyclophosphamide and ifosfamide in the mouse using UPLCESI-QTOFMS-based metabolomics. Biochem Pharmacol.,80(7):1063-1074.

15. Sakr, S.A. and Hany A. Abdelsamie (2016).Effect of ginko biloba extract on cyclophosphamide-induced nephrotoxicity and oxidative stress in albino rats. Canadian J. Pure Appl Sci. 10(2): 3835-3845.

16. Turk G., Eribas A., Sakin F., Sonmez M., Ates S., Ahin A.(2010): Antiperoxidative and anti-apoptotic effects of lycopene and ellagic acid on cyclophosphamide-induced testicular lipid peroxidation and apoptosis. Reprod. Fertil. Dev. ;22:587-596

17. Naeem M Rabeh, Alaa O Aboraya.(2014): Hepatoprotective effect of Dill (Anethum graveolens L.) and fennel ( Foeniculum vulgare) oil on hepatotoxic rats. Pak. J. Nut.13(6):303-309.

18. Ceribasi AO, Turk G, Sonmez M, Sakin F, Atessahin A.(2010): Toxic effect of cyclophosphamide on sperm morphology, testicular histology and blood oxidant antioxidant balance, and protective roles of lycopene and ellagic acid. Basic and Clinical Pharmacology and Toxicology. 107(3):730-736.

19. Hus SM, Raine L, Franger H. (1981): Use of Avidin-biotin peroxidase complex $(\mathrm{ABC})$ in immunoperoxidase techniques: A comparison of $\mathrm{ABC}$ and unlabeled antibody (PAP) procedure. J. Histochem. Cytochem.;29(4):577-580.

20. Henry, R.J. (1974): Creatinine Measurements with Colorimetric Method. Clin Chem Principles and Techniques. Harper\& Row Publishers.

21. Patton, C.J. and Crouch, S. R. (1977): Spectrophotometric and kinetics investigation of the Berthelot reaction for the determination of ammonia. Anal Chem., 49: 464-469.

22. Estakhri, R.; Hajipour, B.; Majidi, H. and Soleimani, H (2013): Vitamin E ameliorates cyclophosphamide induced nephrotoxicity. Life Sci J., 10(6s):308-313.

23. Abraham, P. and Rabi, S. (2009): Nitrosative stress, protein tyrosine nitration, PARP activation and NAD depletion in the kidneys of rats after single dose of cyclophosphamide. Clin Exp Nephrol., 13: 281-287.

24. -Kelman,Z. (1997). PCNA: structure, functions and interactions. Oncogen, 14: 629-640.

25. Romih R, Koprivec D, Štiblar-Martinčič D, Jezernik K.(2001): Restoration of the rat urothelium after cyclophosphamide treatment. Cell Biol Int. 25:531-537

26. Elmore S. (2007). Apoptosis: a review of programmed cell death.Toxicol Pathol.;35(4):495-516.
27. Porter, A.G. and Janicke, R.U. (1999): Emerging roles of caspase-3 in apoptosis. Cell Death Differ. 6: 99-104

28. Sultan, A. and Ayman M. Mahmoud. (2016): GammaGlutamylcysteine Ethyl Ester protects against cyclophosphamide-induced liver injury and hematologic alterations via upregulation of PPAR and attenuation of oxidative stress, inflammation, and apoptosis. Oxidative Medicine and Cellular Longevity 1-14.

29. Groma, V.;Marcussen, N.and Olsen, S. (1997):Aquantitative immunohistochemical study of the expression of mesangial alpha-smooth muscle actin and proliferation marker $\mathrm{Ki}-67$ in glomerulonephritis in man. Virchows Archives, 431: 345350.

30. Ludeman S M. (1999): The chemistry of the metabolites of cyclophosphamide. Curr Pharm .5:627-43

31. -Kern JC, Kehrer JP.(2002). Acrolein-induced cell death: a caspase-influenced decision between apoptosis and oncosis/necrosis. Chem Biol Interact. 139:79-95.

32. Arumugam N, Sivakumar V, Thanislass J, Devaraj H.(1997) Effects of acrolein on rat liver antioxidant defense system. Indian J Exp Biol 35:1373-4

33. Bhatia A L, Manda K, Patni S, Sharma A L. (2006): Prophylactic action of linseed (Linum usitatissimum) oil against cyclophosphamide induced oxidative stress on mouse brain. J Med Food. 9:21-264

34. Stankiewicz, A, Skrzydlewska E, Makieła M. (2002). Effects of amifostine on liver oxidative stress caused by cyclophosphamide administration to rats. Drug Metabol Drug Interact. 19(2):67-82

35. Al-Masri,S. and Ali, W.(2013).The Ameliorating Effects of Fennel Powder, Extract and Oil on Gentamicin Induced Nephrotoxicity in Rats. J Am Sci 9(10):20- 25

36. Al-Amoudi, W.M. (2016): Protective effects of fennel oil extract against sodium valproate-induced hepatorenal damage in albino rats. Saudi Journal of Biological Sciences, 24(4): 915-924

37. Ibrahim, A. A. E. (2007): Does fennel oil have the potentials against apoptosis in testes of rats exposed to mainstream tobacco smoke? Egyptian Journal of Medical Science 28(2): $767-783$

38. Singh, G.; Maurya, S.; de-Lampasona, M.P. and Catalan, C. (2006): Chemical constituents, antifungal and antioxidative potential of Foeniculum vulgare volatile oil and its acetone extract. Food Control, 17(9): 745-752.

39. Sheweita SA, El-Hosseiny LS, Nashashibi MA (2016) Protective Effects of Essential Oils as Natural Antioxidants against Hepatotoxicity Induced by Cyclophosphamide in Mice. PLoS ONE11(11): e0165667. doi:10.1371/journal. pone. 0165667

40. Sakr A. Saber, Shalaby Y. Somaya and Beder H. Rawan (2017). Ameliorative Effect of Fennel Oil on Cyclophosphamide Induced Hepatotoxicity in Albino Rats : British Journal of Pharmaceutical Research 17(2): 1-12, 2017. 